\title{
Research on Cognitive Semantic Analysis of "by-XX" in Network Chinese Passive Sentences
}

\section{Fen Wang}

\author{
Handong Agriculture and Engineering University, Jinan, Shandong, 250100
}

Keywords: by-XX, cognitive semantics, conceptual integration

\begin{abstract}
In recent years, with the popularity of the Internet, people have increasingly used online words for convenience and fun. "by-XX" in the passive Chinese sentence of the Internet is a typical phenomenon of Chinese evolution. The interpretation of this new sentence pattern breaks through the tradition involves complex cognitive semantics, not only to understand the semantic features of the traditional "be" sentence, but also to understand the semantic information contained in "by- $\times x "$. This paper intends to summarize the semantic cognitive structure and the relationship between grammar and semantics by summarizing and analyzing the use of the quotation "by- $x \times$ " in recent years.
\end{abstract}

\section{Introduction}

The popularity of the Internet, coupled with the development of network technology, has also brought about changes in people's language use to a certain extent. The rise of the network language has further enriched people's language systems and led the development of language towards a more diversified direction. In recent years, with the linguistic structure of the Internet term "xxxx", such as "employed", such language forms have sprung up. In TV media and news newspapers, you can see "growth", "being married", "being apologized", "being pregnant", "being strengthened", "being prosperous", "being suicide", "being well-off", "being talked", "being donated", etc. This phenomenon immediately aroused the research interest of sociologists and linguists. It is unreasonable to explain the emergence of such language structures according to conventional linguistic knowledge, because "being" is a passive mark in modern Chinese, and the words following the traditional modern Chinese grammar rules cannot be intransigent. Verbs, which can be matched with them, must be transitive verbs. So it seems that some of the collocations mentioned above are unreasonable and do not conform to the logic of the language. So how did this linguistic phenomenon come about? The research on its production has also aroused the interest of scholars.

\section{The "by- $x x "$ Institution Developed from the Traditional "Be" Sentence}

The times are developing with each passing day, and the language is also changing and reforming under the development of the times. The change in language is also closely related to the changes in people's current life. The popularity and use of the Internet has led to a new language approach, which is the rise of online language. The new type of network language structure "by XX" was also developed from the traditional "being" sentence. The word "be" is also an important issue in linguistics. In modern Chinese, the predicate verbs are dispositional transitive verbs, and some are psychological verbs and cognitive verbs. The original meaning of the word "be" means that the subject is compelled to accept certain actions initiated by the actor. The relationship between the actor and the actor is the transitive relationship, so the part of the suffix following the word "be" must be a transitive verb. In this way, a true "being" sentence can be constructed. However, the emergence of the Internet term " $\mathrm{x} \times$ " is entirely an evolution of the traditional "being" sentence. Words like "being a small three" and "hot pot" are actually nouns. This situation is due to the fact that The latter of " $x \times$ " is a very hot word in the current language environment. Expression in the real context has some special meaning and exaggerates the importance of the latter. There is also a kind of linguistic expression of "being well-off" and "harmonized" is closely related to some 
social events in today's society. Words such as "well-off" and "harmony" have special symbolic meanings in the present. Some political events and historical events in the society can often cause changes in the meaning of linguistic signs, which makes the linguistic symbols derive new meanings. In fact, this new language structure expression also adapts to the new requirements of current language and social development.

\section{The Cognitive Semantic Features of the Online Chinese Passive Sentence "by- $x$ "}

The biggest function of language is communication, and language has some symbolic meaning. Semantics is not a level and independent of grammar research. There is a special relationship between grammar and semantics. This special relationship is also the mapping relationship. If you understand it according to two levels, in simple terms, the grammar is related to the grammatical structure, and the semantics is related to the symbolic meaning of the words formed by the structure. But one thing to note is that the phrase "I eat" is in line with the grammatical rules, but "something eats me" is obviously a sentence that conforms to the grammatical rules, but in grammar this sentence is not in accordance with the rules. . The linguistic structure expression of the network language "by XX" is definitely not a simple copy of the traditional "be" sentence but a linguistic feature with its unique linguistic structure and semantic features. In the context, it is the most able to analyze the cognitive semantic analysis of the network term " $x \times$ ", because the diversity of the expression of this language structure can only be explained in the context.

The word "being" acts as a passive marker to illustrate the relationship. From the syntactic structure point of view, the " $x \times$ " phrase still has many characteristics of "being", but from the syntactical point of view, the form of " $x \times$ " appears as a whole, and the follower of the action will Omitted. Compared with the traditional "being" sentence, the network term "by XX" structure is also the subject of the victim, such as "I graduated today, but at the same time I am also employed", the phrase "I "It is the victim.

\section{The Semantic Analysis of the Passive Chinese Sentence "by-XX"}

After the above discussion, we have also compiled many examples of the passive Chinese sentence " $x \times$ ", and found that the "acceptance of justice" extended from ancient Chinese is deeply reflected in " $\times \times$ ”. For example, "Today's scholarship selection, have you been 'poor and rich?"" "I drove on the road yesterday, there is a feeling of 'the feeling of being "spring"." In fact, the "being" here has the meaning of suffering. The victims of the previous sentence and the latter sentence are "you." The previous sentence can be understood as suffering from the differentiation of the rich and the poor, and the latter sentence is the treatment of the traffic jam in the Spring Festival. Both sentences contain the righteousness, so where does this suffering come from? In fact, as early as in ancient Chinese, there was expression of this meaning. For example, in an article in Pu Songling's "Strange Tales from a Lonely Studio", "Smell your wife's words, such as being snow and ice". This word "being" is followed by the noun phrase. The role of "be" is here is the predicate verb, and "ice and snow" constitutes a verb-object structure, which can be translated as suffering a blizzard. In fact, this example is endless in ancient Chinese. The famous linguist Wang Li said in the book "Ancient Chinese" that "the word is used as a verb, and there is another meaning, that is, the meaning of 'accepting' 'to suffer'." Therefore, we can think of the network. The expression of the linguistic structure of the Chinese passive sentence "by XX" is derived from ancient Chinese. Although the development of language is advanced, it is also a diachronic development. The word "be" was once an expression of "suffering". Although the words "conceived" have gradually been forgotten in the development of history, the meaning of verbs has gradually blurred, but now it has re-emerged in the network language.

"Besides XX" means not only expressing "wearing" or "experiencing", but also expressing the meaning of coercion. This "XXXX" network Chinese expression, which expresses the willingness to force, is also common in ancient Chinese, expressing a "strong sense" that is not controlled by human beings but by external forces. For example, in the current environment, college graduates are 
'researched for 'research' and 'public examinations' and "thousands of students encounter 'by credit card' has owed 100 yuan annual fee" This is not my will, but is forced by external forces. This kind of being forced is forced by external forces. From the above, it can be known that "being examined by the doctor", "being publicly tested" and "by credit card" are not the subjective will of the speaker. The behavior here has the meaning of "have", among which The implementer was also saved. But from the context here, we can conclude that the actor is a collective unit, not figurative but abstract.

Regarding the expression of the linguistic structure of the passive Chinese sentence "XXXX", more examples express a meaning that is recognized. For example, some phrases are "winning", "being married", "being pregnant" and so on. To a certain extent, this "expressed by $\mathrm{XX}$ " language structure is more popular in the popular language. Even if the actor is omitted, it can be understood according to the language environment of the sentence and the entire context. The meaning of the sentence, understand its semantic rules and grammatical structure. In a nutshell, this type of structural model is also meant to contain a certain amount of compulsion. This "identified" can only allow the passive to passively accept this bad result, which is also a forced meaning.

\section{Conclusion}

With the development of the times, modern Chinese is also constantly enriched, optimized and perfected. The emergence of " $x \times$ " in the passive Chinese sentence of the Internet is born out of the ordinary. This language structure expression has the meaning of "wearing righteousness", "forced righteousness", "recognition", etc., all of which are the integration and application of different semantic information. It is also the development process of "lexicalization".

\section{References}

[1] Zhang Keding. Exploration of the Derivation of Linguistic Signs [J].Journal of PLA University of Foreign Languages, 2001, (6).

[2] Xiong Xueliang, Wang Zhijun. A Cognitive Comparative Analysis of English and Chinese Passive Sentences [J]. Foreign Languages Journal, 2001, (3). 